Chemometrics and Intelligent Laboratory Systems, 16 (1992) 77-86

Elsevier Science Publishers B.V., Amsterdam

\title{
Neural networks used as a soft-modelling technique for quantitative description of the relation between physical structure and mechanical properties of polyl ethylene terephthalate) yarns
}

\author{
A.P. de Weijer ${ }^{1}$, L. Buydens and G. Kateman \\ Department of Analytical Chemistry, Catholic University of Nijmegen, Nijmegen (Netherlands) \\ H.M. Heuvel \\ AKZO Research Laboratories Arnhem, P.O. Box 9300, 6800 SB Arnhem (Netherlands)
}

(Received 1 September 1991; accepted 30 December 1991)

\begin{abstract}
De Weijer, A.P., Buydens, L., Kateman, G. and Heuvel, H.M., 1992. Neural networks used as a soft-modelling technique for quantitative description of the relation between physical structure and mechanical properties of poly(ethylene terephthalate) yarns. Chemometrics and Intelligent Laboratory Systems, 16:77-86.

The use of artificial neural networks (ANNs) to develop a model of the relation between physical structure and mechanical properties of poly(ethylene terephthalate) yarns is investigated. The relation was studied on a set of 295 drawn yarn samples, produced under an exceptionally large variety of applied process conditions. The ANN, consisting of three layers of neurons, was trained using physical structure and property measurements of these 295 yarns as input and output patterns, respectively. All ten properties were fitted to the physical structure within a variance that was acceptable for quantitative use. This paper demonstrates that ANNs may be applied as a quantitative technique in solving a real-world problem. The use of ANNs resulted in an improved understanding of the complicated relation between physical yarn structure and yarn properties.
\end{abstract}

\section{INTRODUCTION}

Correspondence to: Dr. L. Buydens, Department of Analytical Chemistry, Catholic University of Nijmegen, Toernooiveld 1,6525 ED Nijmegen, Netherlands.

${ }^{1}$ Permanent address: AKZO Research Laboratories Arnhem, P.O. Box 9300, 6800 SB Arnhem, Netherlands.
A great deal of interest has recently been displayed in the use of artificial neural networks (ANNs) as a classification technique. A few publications show that ANNs can reach higher numerical accuracy than conventional techniques [1-3]. 
ANNs develop their own internal model, depending on the training set of cxamples. No timc and effort has to be spent on the development of a model framework as is necessary, for instance, for nonlinear regression. This makes ANNs an interesting soft-modelling technique, especially when modelling complex and unknown relations.

A study has been set up to investigate the relation between the physical structure and the mechanical properties of industrial poly(ethylene terephthalate) (PET) yarns. PET fibers are manufactured by extruding molten PET polymer through a number of fine orifices (diameter 300$600 \mu \mathrm{m})$ into an air stream which cools and solidifies the filaments. The polymer chains in these filaments are subsequently oriented and (partially) crystallized by stretching the originally undrawn material (Fig. 1). By varying the spinning and stretching conditions over wide ranges, a set of yarns is obtained with widely differing mechanical properties.

Almost all process conditions can be translated into combinations of temperature, tension, and residence time. Process conditions determine the thermal and mechanical properties of the yarns produced. The relation between process condi-

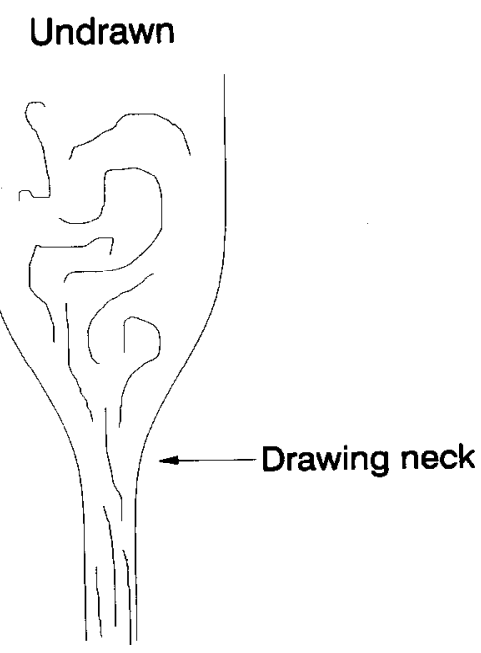

Drawn

Fig. 1. Orientation of the molecules in the drawing process.

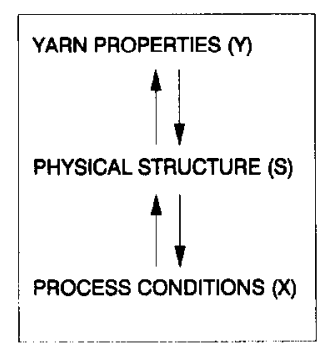

Fig. 2. Intermediate position of the physical yarn structure.

tions and properties is regarded as a complex one, which is highly machine dependent [4]. More detailed information concerning the spinning and drawing process of PET yarns is given in the literature [5].

The need for high-quality yarns in combination with economic yarn spinning processes has led to a growing interest in the study of physical yarn structure. The physical yarn structure can be regarded as an intermediate between process conditions and yarn properties (Fig. 2). A set of process conditions, $\mathbf{X}=\left[x_{1}, x_{2}, \ldots, x_{l}\right]$, results in a physical yarn structure, $\mathbf{S}=\left[s_{1}, s_{2}, \ldots, s_{m}\right]$, which is responsible for the intrinsic yarn properties $\mathbf{Y}=\left[y_{1}, y_{2}, \ldots, y_{n}\right]$.

Qualitative descriptions of the relations between process conditions, physical structure and (mechanical) properties have been developed by Heuvel and Huisman $[4,6]$.

This paper describes the use of an artificial neural network (ANN) as a soft-modelling technique for the relation between physical structure and properties of PET yarns. It should primarily provide an improved understanding of the mechanical yarn properties in terms of the physical structure, which is an important condition for effective process developments as well as effective product development. Secondly, if it is possible to fix this relation unambiguously, it should be possible to predict the properties of various types of PET yarn (different processes, different manufacturers) directly on the basis of their structure.

The choice of using neural networks for the relation described above is that the relation is complex and not known in detail. An adaptive 
self-learning system, like an artificial neural network, is therefore appropriate.

It is sometimes stated that neural networks are somehow inherently inaccurate and therefore best suited to 'fuzzy' qualitative reasoning [7]. A great deal of interest has therefore been displayed in the capabilities of an ANN in performing some kind of qualitative reasoning, as in classification, for instance. Clearly, these developments were aimed at uniting the advantages of human pattern recognition and computer number crunching. Relatively little work has been done on the ability of ANNs in solving quantitative structureactivity relationship (QSAR) problems $[8,9]$. For some applications, neural networks can achieve a higher numerical accuracy than more conventional techniques [1-3]. The availability of a database on PET yarns, in which a great variety of structures and properties are recorded, offered a nice opportunity to test this ability.

\section{THE USE OF NEURAL NETWORKS}

Work on ANNs has a long history. The first steps in ANN research were taken forty years ago. The breakthrough came in 1986, when Rumelhart et al. [10] discovered a learning rule for complex ANNs. Since then, the ANN evolved from a theoretical study towards real-world applications.

ANNs differ from other approaches in the way that they learn from examples. The computer simply runs through the examples again and again and learns from the mistakes it made in previous trials. ANNs are therefore called naive, initially model-deficient, systems. Thcy are not restricted by prior conditions, such as are imposed by nonlinear calculations, for instance.

The basic computational elements are neurons. Each neuron has a series of weighted inputs, $W_{i} X_{i}$, which may be input signals or the output from other neurons. Information, or 'knowledge', is storcd in the connections between these elements in the form of weights $W_{i}$, rather than in the neurons themselves. The simplest neuron sums $N$ weighted inputs and passes the result through a nonlinearity (transfer function)

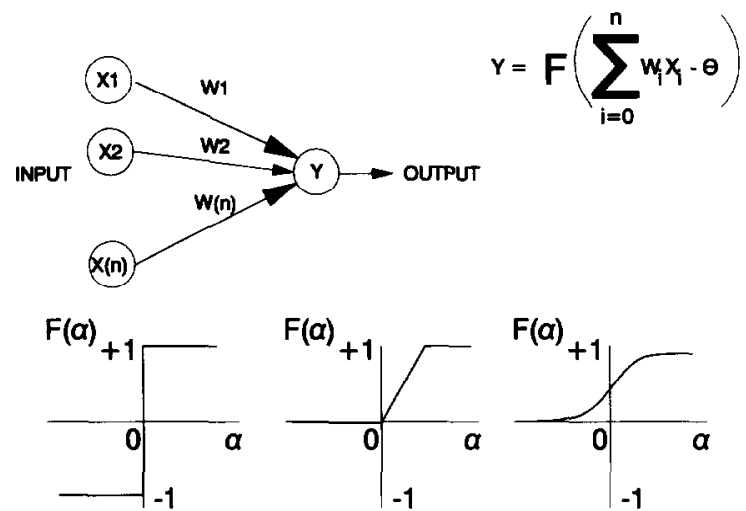

Fig. 3. Neuron which forms weighted sums of $N+1$ inputs and passes the results through a nonlinearity.

as shown in Fig. 3. This result serves cither as an output signal or as an input signal for other neurons. A neuron is characterized by an internal threshold of offset $\theta$ and by the type of nonlinearity.

Learning algorithms for neural networks are usually gradient-descent methods that minimize the mean square of system error iteratively by adapting the weights.

The most widely used type of ANN is the feedforward network. These types of networks are characterized by the feedforward connectivity of an input layer through 'hidden layers' to an output layer (Fig. 4). Feedforward network configurations are primarily trained by backpropagdtion. This training algorithm can find a good set

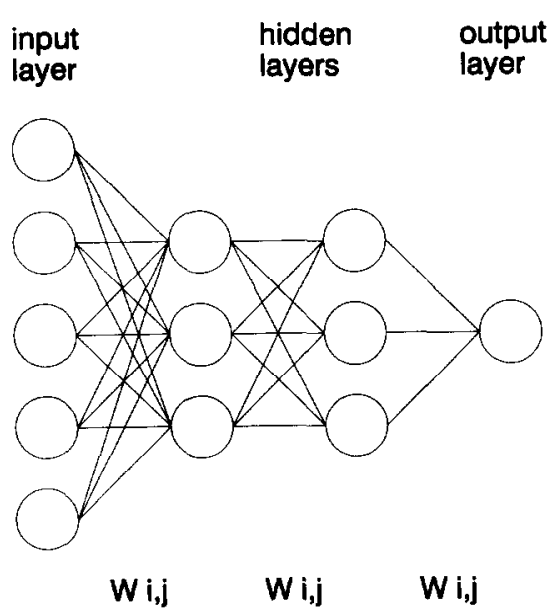

Fig. 4. A feedforward network with two hidden layers. 
of weights and threshold using a gradient-descent method [11].

In the backpropagation learning procedure the adaptation of the internal weights is proportional to a factor $\eta$ (learning rate) and a weight gradient (Eqns. 1 and 2). When the learning rate is set too high, local minima are overlooked during the descent down the error surface. A learning rate which is too low leads to slow convergence to a minimum system error.

When there is no difference between the (measured) target value $\left(t_{j}\right)$ and the (estimated) outpuit value $\left(o_{j}\right)$, so that $t_{j}-o_{j}$ is zero, no adaptation or learning takes place since $\Delta W_{i j}$ is equal to zero.

$E=\frac{1}{2} \sum_{\text {all } j}\left(t_{j}-o_{j}\right)^{2}$

$\Delta W_{i j}=-\eta \frac{\delta E}{\delta w}$

Adaptation of the internal network weights proceeds iteratively, usually until the system error, $E$, is smaller than a termination value, $\alpha$, which is predetermined by the user.

After having learned to establish correlations between input and output vectors, one then applies new, previously unseen inputs to the trained network. The output can be regarded as a prediction drawn by the neural net based on its knowledge of the fundamental relationship between input and output. More general information concerning neural networks can be found in ref. 11 .

In the next two sections we present a detailed discussion of the input and output variables, and the physical structure variables and property variables, respectively.

\section{PHYSICAL YARN STRUCTURE}

Important characteristics of the physical structure are the crystallinity, size and orientation of the crystals and, for the amorphous domains, their size, as well as the orientation of the molecules. Wide-angle X-ray (WAXS) diffraction is used to obtain information about the crystalline morphology (crystal size and crystal packing of

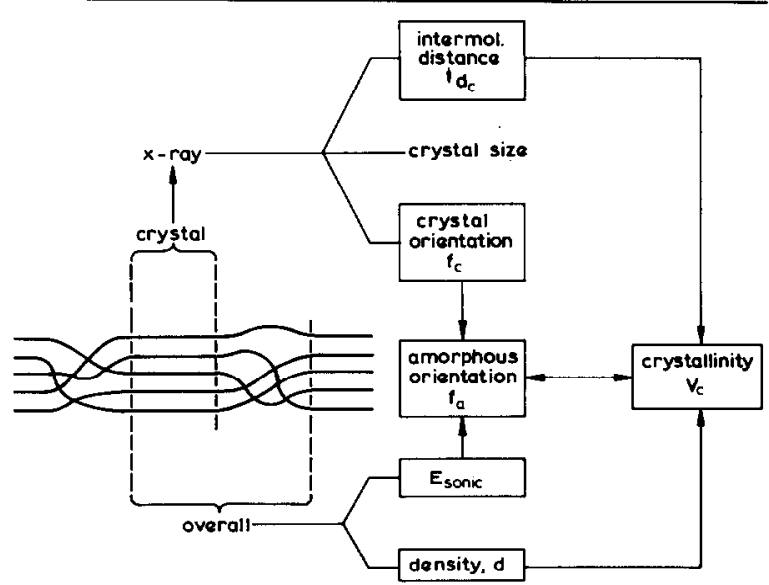

Fig. 5. Schematic representation of a combination of structure characterization techniques.

the molecules in the crystal). The combination of WAXS and overall density measurements yields a measure of the crystallinity. The combined results of pulse propagation, crystallinity and crystalline orientation finally yield an estimate of the amorphous orientation [12]. A schematic survey of the procedure outlined above is given in Fig. 5 .

A general structure model of an oriented PET fibre is given in Fig. 6, which shows that ordered (crystalline) regions alternate with less-ordered (amorphous) domains. Depending upon the pro-

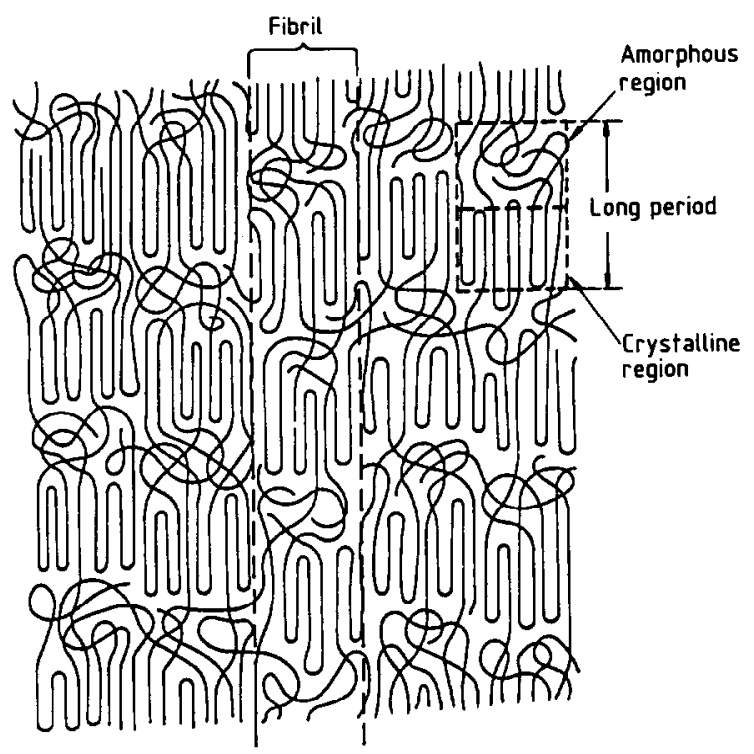

Fig. 6. Two-phase model. 
cess conditions, the polymer molecules are oriented in different ways along the fibre axis, resulting in different structures. Mechanical responses to loading or heating are primarily determined by the amorphous phase. In this study special attention has been paid to the selection of physical structure parameters relating to the amorphous phase. The physical structure of PET yarns was investigated by means of X-ray diffraction, density measurements, sonic pulse propagation and measurements of the birefringence.

The study of the structure-property relation was based on a set of 295 drawn yarn samples, manufactured under a wide variety of applied process conditions. In total, twelve structure quantities were measured. Since correlations exist, a minimum number of structure parameters were chosen - as independent of each other as possible - in order to provide an effective characterization and interpretation of the structure. This was done by subjecting all quantities to a principal component analysis (PCA). On the basis of this analysis the following set of structure parameters $\mathbf{S}_{m}$, has been chosen: 1 . the volume fraction of amorphous material $\left(1-V_{c}\right) ; 2$. the yarn viscosity as a measure of molecular length $\left(\eta_{\text {rel }}\right)$; 3. the amorphous orientation factor $\left(F_{\text {as }}\right)$; 4. the contour length distribution of the tie chains between successive crystals $\left(F_{\mathrm{as}} / F_{\mathrm{ab}}\right) ; 5$. the aver- age volume of the individual amorphous regions $(G)$. Although ANNs are not sensitive to correlations, these five independent structure parameters were selected a priori because they can easily be explained in terms of the two-phase model $[13,14]$.

\section{MECHANICAL YARN PROPERTIES}

The stress-strain curve is very important for characterizing the mechanical behavior of yarns; it contains information concerning tensile strength, energy absorbance, elongation, and modulus.

The textilc yarn propertics werc measured on conditioned yarns using an Instron Type 1121 tensile tester. The data for each yarn are the average values over three repeated recordings of stress-strain curves. Eight properties were determined from the stress-strain curve and its first derivative, of which six were ultimately used. Fig. 7 shows an example of a stress-strain curve (Fig. 7a) and a modulus strain curve (Fig. 7b) of a PET industrial yarn. The properties which were determined from these plots are indicated in the figures.

Dimensional stability is also an important feature in the characterization of yarn properties. (a)

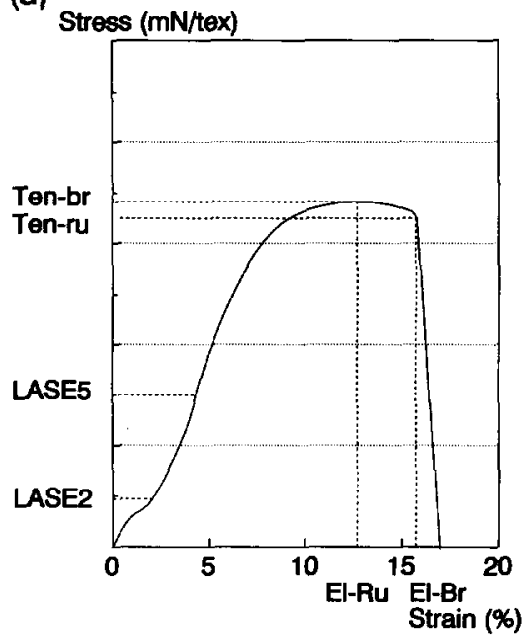

(b)

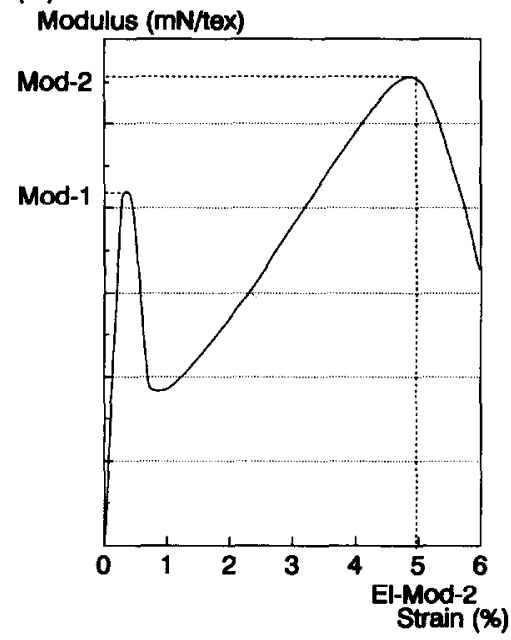

Fig. 7. (a) Stress-strain curve of a PET industrial yarn. (b) Modulus-strain curve of a PET industrial yarn. 
From each of the 295 yarns two shrinkages were measured in hot air under tension at $160^{\circ} \mathrm{C}$ (HAS-160) and tensionless at $190^{\circ} \mathrm{C}$ (SHA-190). Finally, the shrinkage force at $160^{\circ} \mathrm{C}$ (HAST-160) and the residual shrinkage force at room temperature (RHAST-160) were measured.

\section{EXPERIMENTAL}

In order to determine an optimum set of conditions for training a network for this data set, a variety of networks has been trained under a wide range of number of hidden units (1-10), momentum $(0.3-0.9)$ and learning rates $(0.2-1.0)$. The criterion for determining the optimum pdrameter setting was the residual variance of the test set. The property data were scaled between 0.2 (lowest occurring value) and 0.8 (highest occurring value). Scaled outputs are necessary to accomodate the bounded range of the sigmoid output function $(0-1.0)$. The input values were scaled between -1 and 1 .

The type of ANN that was ultimately used is a multilayered, feedforward network (Fig. 8) with five input units, corresponding to the five structure parameters described above (Physical Yarn Structure), one hidden layer, and 10 output units, corresponding with the ten properties mentioned

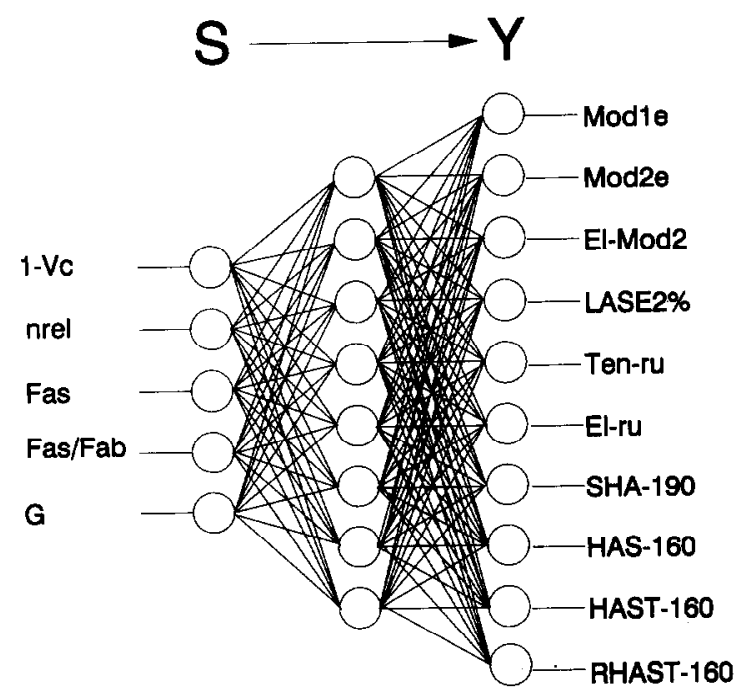

Fig. 8. Schematic representation of the network used.
TABLE 1

Artificial neural network specifications

\begin{tabular}{ll}
\hline Learning rate & 0.5 \\
Momentum & 0.8 \\
Transfer function & Sigmoid \\
Termination & Min. error in test set \\
No. input neurons & 5 \\
No. hidden neurons & 8 \\
No. output neurons & 10 \\
Input scaling (min/max) & $(-1 / 1)$ \\
Output scaling (min/max) & $(0.2 / 0.8)$ \\
\hline
\end{tabular}

in the section on Mechanical Yarn Properties. The bias weights are implemented in the nodes. By training the ANN with several numbers of hidden units and by comparison with the prediction errors of a test set, the optimum number of hidden units was determined to be eight. A backpropagation training algorithm for multilayered perceptron networks [10] has been written in Turbo Pascal. The specifications for the neural network and the reverse propagation training algorithm are summarized in Table 1.

In contrast to, for instance, adaptational learning concerning classification, in which the output patterns of the training set are noiseless factors *, quantitative measurements in QSAR learning contain, in addition to 'true' output values, both noise and systematic errors. The fitting of outliers and noise in the training set leads to poor predictions and it is essential that the criterion for optimality is a situation in which no fitting of noise and outliers is present. To achieve this, the following technique has been used. The data set containing the structures and properties of 295 drawn PET yarns was divided into two subsets. A training set of 225 yarns was randomly subtracted from the data set. The remainder, 70 yarns, was selected for participation in a test set. During the reverse propagation training procedure, the training set and the test set were continuously evaluated in terms of model accuracy. Only the error

\footnotetext{
* The output patterns in the training set are classified correctly or not. The noise level is discrete in classification. Discrete and continuous (e.g. Gaussian) noise levels require different validation methods for the results obtained.
} 
$E=\frac{1}{2} \Sigma\left(t_{j}-o_{j}\right)^{2}$ of the training set was used to adapt the internal network weights. A minimum error in the test set is used as a primary termination criterion. If the system is trained beyond this minimum, the system errors of the training set will decrease further because the fitting noise of the training set leads to smaller system errors. The system error with respect to the test set will increase because noise in the randomly subtracted test set is likely to be different from the fitted noise of the training set. This phenomenon is known as overtraining and is described in ref. 9. If, after a predetermined number of iterations, no minimum in the error of the test set is found, the learning procedure is also terminated.

\section{RESULTS AND VALIDATION OF THE TRAINED ANN}

A neural network for the relation between the structure and properties of PET yarns was trained in the way described above. The ability of the network to estimate the measured properties from the PET experiment is tested by plotting all mcasured properties against all propertics cstimated by the trained network. This is illustrated for the 'tenacity at rupture' property in Fig. 9. A comparison has been made between the perfor-

Estimated Tenacity at rupture ( $\mathrm{mN} / \mathrm{Tex})$

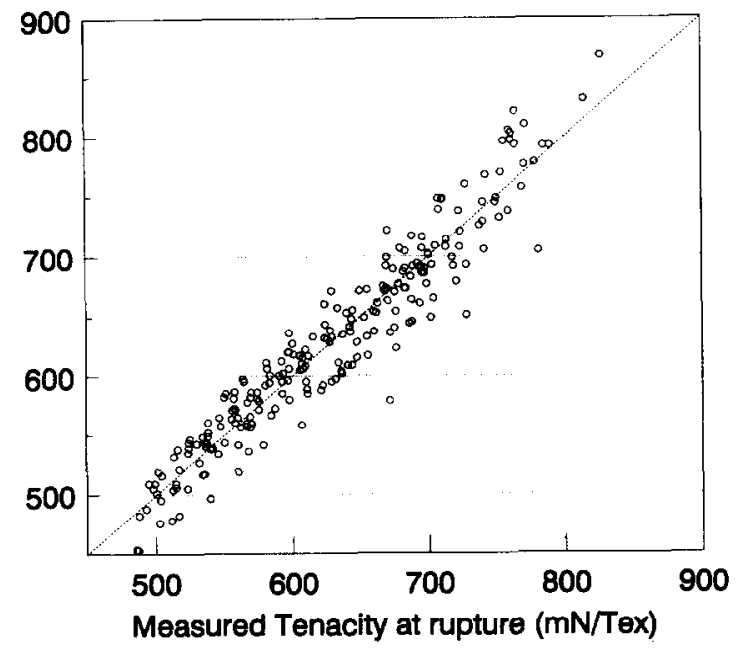

Fig. 9. Measured tenacity at rupture vs. estimated tenacity at rupture for training set. mance of ANN and two other multivariate techniques: principal component regression (PCR) and partial least squares (PLS). The relative standard deviation of the error, $s_{\text {res }}$ was taken as a measure of the residual error.

The relative residual standard deviations of the estimation of the training set, $s_{\text {res }}$, were determined with respect to all properties in the training set used according to the following formula:

$s_{\text {res }}=\frac{\sqrt{\sum_{\text {training set }}\left(\mathbf{Y}_{\text {meas }}-\mathbf{Y}_{\text {est }}\right)^{2}}}{(k-1)} * \frac{100 \%}{\mathbf{Y}_{\max }-\mathbf{Y}_{\min }}$

in which $\mathbf{Y}_{\text {meas }}$ is the measured value of property $Y$ and $Y_{\text {est }}$ is the estimation given by the neural net for $Y_{\text {meas }} . Y_{\text {max }}$ and $Y_{\text {min }}$ are the maximum and the minimum value of measured property $Y$ in the training set, respectively. $k$ is the number of yarns in the training set. Since we do not know how to determine the degrees of freedom for an ANN we assumed $k-1$ degrees of freedom for the standard deviation.

The residual variance, $s_{\text {res }}^{2}$, is composed of two independent variances. (1) It depends on the pure measurement error in the properties $\mathbf{Y}_{\text {meas }}$. These variances, $s_{\text {pe }}^{2}$, were determined from three repeated recordings of each property during laboratory measurement. (2) The second component is the model variance, which is composed of a lack of fit, $s_{\text {lof }}^{2}$, and a variance resulting from propagated pure errors of the structure as determined by laboratory measurements.

Table 2 shows the variance estimations, $s_{\text {res }}^{2}$, of all properties for the ANN and for PCR for the training set. This table also summarizes $s_{\mathrm{pe}}^{2}$ and $s_{\text {res }}^{2}$ of the test set for all ten properties. As shown in this table the residual variances of all properties of the ANN are smaller than the variances obtained by PCR. The results for the calibration of the data set with PLS did not differ from the PCR results.

The results also indicate that there is still a significant model error of the ANN with respect to the data. It is not possible to determine whether this is due to a significant lack of fit, since we do 
TABLE 2

Relative variance of residuals for $\mathrm{ANN}$ and PCR, and relative variance of pure error in $Y$

\begin{tabular}{llrrrr}
\hline Property & $\begin{array}{l}\text { ANN } \\
\text { test set, } \\
s_{\text {res }}^{2}\end{array}$ & $\begin{array}{l}\text { ANN, } \\
s_{\text {res }}^{2}\end{array}$ & $\begin{array}{l}\text { PCR } \\
\text { test set, } \\
s_{\text {res }}^{2}\end{array}$ & \multicolumn{1}{l}{$\begin{array}{l}\text { PCR, } \\
s_{\text {res }}^{2}\end{array}$} & \multicolumn{1}{c}{$s_{\text {pe }}^{2}$} \\
\hline SHA-190 & 14.4 & 9.4 & 21.2 & 33.1 & 1.4 \\
HAS-160 & 14.5 & 9.5 & 20.8 & 37.6 & 2.0 \\
IIAST-160 & 23.2 & 18.0 & 52.1 & 45.3 & 6.3 \\
RHAST-160 & 31.8 & 22.6 & 55.3 & 48.8 & 8.4 \\
Mod-1 & 30.5 & 20.1 & 63.2 & 49.9 & 23.0 \\
Mod-2 & 31.1 & 20.3 & 103.5 & 87.4 & 6.3 \\
El-Mod-2 & 30.9 & 27.2 & 142.5 & 173.7 & 1.7 \\
LASE2\% & 21.1 & 17.4 & 51.1 & 48.6 & 10.9 \\
Ten-Ru & 20.3 & 18.3 & 57.7 & 60.5 & 6.3 \\
El-Br & 109.1 & 77.7 & 184.0 & 301.0 & 38.4 \\
\hline
\end{tabular}

not know how to calculate the share of propagated errors in the model error. More research is necessary to discover better approximations for the induced errors and the calculation of lack of fit for neural networks. However, the residual variances of all properties were small enough for the ANN to be used in practical work.

INTERPRETATION OF RESULTS IN TERMS OF THE TWO-PHASE MODEL

A factorial design was applied to the trained and validated network, and the influences of each structure parameter on each property were determined. The results of this experiment turned out to be useful in achieving an improved understanding in this interesting relation.

The five structure parameters resulted in a $2^{5}=32$ experiment design. This experiment was not possible without the use of a model for the structure-property relation since the structure of PET is determined by the process conditions in a complicated and intercorrelated manner. It is therefore not possible to produce 32 yarns in which all possible combinations of high and low values of structure parameters are present. For the neural network factorial design experiments, the lowest and highest values were equal to the minimum and maximum structure values found in the training set, respectively. Thus, only interpolated estimations of the neural network were used for the factorial design. In this way, the main effects of structure parameters on properties could be estimated. Table 3 summarizes the results of all contributions.

For instance, the fraction of amorphous material $\left(1-V_{c}\right)$ exerts a principal effect of $-9.3 \%$ on the tenacity at rupture (Ten-Ru). The sign of the contribution shows in this case that a high fraction of amorphous material results in low tenacities. Table 3 also shows that not all of the variation in properties is solely due to the pure influence of the structure parameters. The variation of Ten-Ru is only $70 \%$ accounted for by the pure influences; consequently, $30 \%$ of this parameter is due to higher-order and cross terms. Although the relations between structure and properties are not linear, the results of the factorial design

TABLE 3

Main effects of structure parameters $S_{m}$ on property parameters $Y_{n}$ of PET yarns

\begin{tabular}{|c|c|c|c|c|c|c|}
\hline Property & $\begin{array}{l}1-V_{c} \\
(\%)\end{array}$ & $\begin{array}{l}\eta_{\text {rel }} \\
(\%)\end{array}$ & $\begin{array}{l}F_{\text {as }} \\
(\%)\end{array}$ & $\begin{array}{l}F_{\mathrm{as}} / F_{\mathrm{ab}} \\
(\%)\end{array}$ & $\begin{array}{l}G \\
(\%)\end{array}$ & $\begin{array}{l}\text { Percentage } \\
\text { explained }\end{array}$ \\
\hline SHA-190 & 15.2 & -0.2 & 24.3 & -9.3 & -17.6 & 67 \\
\hline HAS-160 & 14.7 & -1.6 & 33.5 & -8.0 & -15.7 & 74 \\
\hline HAST-160 & 6.5 & -5.4 & 55.2 & 1.4 & -3.6 & 72 \\
\hline RHAST-160 & 7.5 & -7.5 & 47.4 & 2.9 & -0.5 & 68 \\
\hline Mod-1 & -13.5 & 7.4 & 51.3 & -3.8 & -4.8 & 81 \\
\hline Mod-2 & -6.1 & 8.4 & 55.1 & -5.6 & -9.4 & 85 \\
\hline El-Mod-2 & -13.8 & 10.4 & -31.6 & 1.1 & -1.2 & 59 \\
\hline LASE $2 \%$ & -11.6 & 2.5 & 58.5 & -0.1 & -0.5 & 74 \\
\hline Ten-Ru & -9.3 & 11.6 & 20.0 & -11.3 & -17.1 & 70 \\
\hline $\mathrm{El}-\mathrm{Br}$ & 9.9 & -5.1 & -44.6 & 7.9 & -0.8 & 69 \\
\hline
\end{tabular}


are good enough for qualitative interpretation. For quantitative results, like prediction, the direct use of the trained ANN is necessary.

All contributions in Table 3 could be explained in terms of the two-phase model. A paper describing this model has been accepted for publication [14].

\section{USING A TRAINED ANN FOR PREDICTION}

If the physical structure of PET yarns is unambiguously fixed by the five physical structure parameters, as described above, it should be possible to predict the mechanical properties of PET samples directly on the basis of their physical structure, using a trained and validated neural network. To test the performance of the neural network on the prediction of mechanical properties from physical structure, a set of 30 previously unseen yarns was selected. The structures and properties of all the yarns were measured. The prediction set consistcd of PET yarns manufactured in processes other than the process used to produce the yarns from the training set and the test set. The set was characterized by a great diversity of structures. Fig. 10 shows the results of

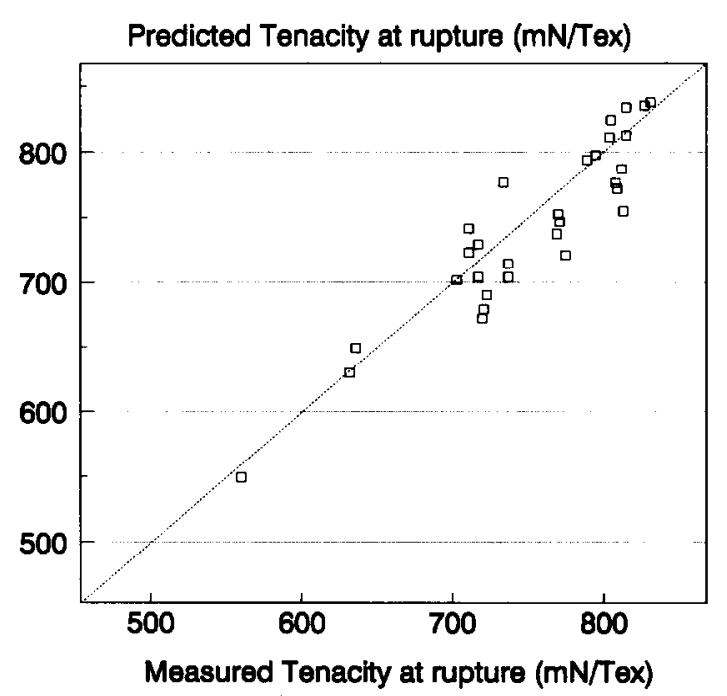

Fig. 10. Measured tenacity at rupture vs. predicted tenacity at rupture of 30 previously unseen PET yarns. The prediction is performed by a neural network, trained for the $S_{m} \rightarrow Y_{n}$ relation.

\section{TABLE 4}

Comparison of neural network performance in estimation of training set, test set and prediction set, expressed in a relative standard deviation of residuals

\begin{tabular}{llll}
\hline Property & $\begin{array}{l}s_{\text {train }} \\
(\%, k=225)\end{array}$ & $\begin{array}{l}s_{\text {test }} \\
(\%, k=70)\end{array}$ & $\begin{array}{l}s_{\text {pred }} \\
(\%, k=30)\end{array}$ \\
\hline SHA-190 & 3.1 & 3.8 & 5.8 \\
HAS-160 & 3.1 & 3.8 & 6.1 \\
LASE2\% & 4.2 & 4.6 & 7.3 \\
Ten-Ru & 4.3 & 4.5 & 5.4 \\
\hline
\end{tabular}

the prediction plotted against the measured valucs of the tenacity at rupture for all 30 yarns.

The standard deviation of error is defined as

$$
\begin{aligned}
s_{\text {pred }}= & \frac{\sqrt{\sum_{\text {prediction set }}\left(\mathbf{Y}_{\text {meas }}-\mathbf{Y}_{\text {pred }}\right)^{2}}}{(k-1)} \\
& * \frac{100 \%}{\mathbf{Y}_{\max }-\mathbf{Y}_{\min }}
\end{aligned}
$$

In which $Y$ is the measured value of property $Y$ and $Y_{\text {pred }}$ is the prediction of the neural net for $Y$. $Y_{\text {max }}$ and $Y_{\min }$ are the maximum and the minimum value of property $\mathbf{Y}$ measured in the training set, respectively. $k$ is the number of yarns in the prediction set. The standard deviations of the residuals are shown in Table 4 as a measure of the performance of the network in estimating and predicting mechanical properties on the basis of physical structure.

Since the standard deviation of the prediction set is not significantly different from the standard deviation of the test set, it is concluded that this ANN permits the prediction of the properties of yarn on the basis of a minimum set of five structure parameters. The underlying relations have proved to be almost independent of the production process applied.

\section{FUTURE WORK}

In order to gain more insight into the influences of process parameters on the physical structure of PET samples, we are now using an ANN to calibrate this relation. Preliminary re- 
sults indicate that it is possible to simulate this relation with high accuracy.

Another interesting relation for process and product development is the reverse relation of the one discussed, viz. the relation between mechanical properties and physical structure. From an industrial point of view it is interesting to know which structures are possible for a combination of mechanical propertics. Since this is an ambiguous relation, it is not possible to use a neural network for this reverse relation. Therefore an optimization technique, a genetic algorithm (GA), is combined with the trained neural network to discover optimum structures in the solution space. Evolutionary search procedures have been succesfully applied as a learning algorithm for neural networks [15,16]. Preliminary experiments have demonstrated that the combination of the ANN described in this paper with a GA is a useful tool in product and process development. The results of our work using this symbiosis will be published later.

We have demonstrated a real-world application of a neural network applied to a large and complex problem. Our work shows that neural networks, as a naive approach, are suited to the mapping of quantitative, nonlinear relations. The neural network approach is a generally applicable method for the solution of problems having previously unknown search spaces. The question of whether ANNs can compete with other modelling techniques, such as PLS or PCR, can only be answered conclusively after profound research on validation methods for ANNs. Research in this area is continuing and will be presented in future papers.

\section{ACKNOWLEDGEMENTS}

The authors wish to acknowledge the following contributions to the work presented: Akzo Research Laboratories for supplying the data and for their support of this work and A. Smits, C.B. Lucasius and $M$. Derksen for providing stimulating and fruitful ideas.

\section{REFERENCES}

1 A. Lapedes and R. Farber, Nonlinear signal processing using neural networks, LA-UR87-2662, Los Alamos National Library, 1987.

2 J.R. Long, V.G. Gregoriou and P.J. Gemperline, Spectroscopic calibration and quantitation using artificial neural networks, Analytical Chemistry, 62 (1990) 1791-1797.

3 T. Aoyama, Y. Suzuki and H. Ichikawa, Neural networks applied to quantitative structure-activity relationship analysis, Journal of Medicinal Chemistry, 33 (1990) 25832590.

4 R. Huisman and H.M. Heuvel, The effect of spinning speed and drawing temperature on structure and properties of poly(ethylene terephthalate) yarns, Journal of $A p$ plied Polymer Science, 37 (1989) 595-616.

5 R.W. Moncrieff, Man-Made Fibres, Heywood Books, London, 1969.

6 H.M. Heuvel and R. Huisman, Infrared spectra of poly(ethylene terephtalate) fibers. Fitting of spectra, evaluation of parameters, and applications, Journal of Applied Polymer Science, 30 (1985) 3069-3093.

7 R. Hecht-Nielsen, Neurocomputing: picking the human brain, IEEE Spectrum, 4 (1988) 36-41.

8 V.S. Rose, R.M. Hyde and H.J.H. Macfie, U.K. usage of chemometrics and AI, Journal of Chemometrics, 4 (1990) $355-360$

9 J. Zupan and J. Gasteiger, Neural networks: A new method for solving chemical problems or just a passing phase?, Analytical Chimica Acta, 248 (1991) 1-30.

10 D. Rumelhart and J. McClelland, Parallel Distributed Processing, Vol. 1, M.I.T. Press, Cambridge, MA, 1986.

11 D. Rumelhart, G. Hinton and R. Williams, Learning representations by back-propagating errors, Nature, 323 (1986) 533-536.

12 R. Huisman and H.M. Heuvel, Effect of winding speed on the physical structure of as-spun poly(ethylene terephthalate) fibers, including orientation-induced crystallisation, Journal of Applied Polymer Science, 22 (1978) 2229-2243.

13 H.M. Heuvel, Internal Report CDSF9006, Akzo Research Laboratories Arnhem, Arnhem, 1990.

14 H.M. Heuvel, L.J. Lucas, C.J.M. van den Heuvel and A.P. de Weijer, Experimental relations between physical structure and mechanical properties of a huge number of drawn poly(ethylene terephthalate) yarns, Journal of $A p$ plied Polymer Science, in press.

15 D.J. Montana and L. Davis, Training feedforward neural networks using genetic algorithms, Machine Learning, 4 (1990) 762-767.

16 R.K. Belew, J. McInerney and N.N. Schraudolph, Evolving networks: using the genetic algorithm with connectionist learning, CSE Technical Report No. CS90-174, Cognitive Computer Science Research Group, University of California, San Diego, CA, 1990. 